\title{
Nonhuman primate models for SARS-CoV-2 research: Consider alternatives to macaques
}

\author{
The SARS-CoV-2 pandemic has significantly increased the demand for rhesus macaques, which might outstrip \\ the supply for COVID-19 research and for other biomedical research studies. Baboons, another well-characterized \\ research primate model, should be considered as a promising alternative for COVID-19 disease studies.
}

\author{
Michael C. Chang, Sheri Hild and Franziska Grieder
}

I he National Institutes of Health (NIH) supports multiple nonhuman primate colonies, including colonies housed at the National Primate Research Centers (NPRCs) and other primate facilities, such as the Keeling Center for Comparative Medicine and Research Specific-Pathogen-Free Baboon Research Resource and the Caribbean Primate Research Center. These centers and facilities are supported through grants and cooperative agreements administered by the Office of Research Infrastructure Programs (ORIP) within the NIH Office of the Director. These ORIP-supported nonhuman primate resources provide high-quality, well-characterized rhesus macaques, baboons, African green monkeys/vervets, and other primates for use in biomedical research across scientific disciplines, with studies supported by Institutes, Centers and Offices across the NIH.

Due to their genetic, physical and physiological similarities to humans, nonhuman primates are often the best models for human disease research, particularly when human studies are not ethical or feasible. Nonhuman primates have played a key role in helping scientists to understand and develop treatments for a variety of infectious diseases, such as AIDS, Zika virus and, most recently, SARS-CoV-2 infection/COVID-19. Nonhuman primates also have been used to determine the safety and efficacy of vaccines, devices, and therapies before their use in humans, including testing of the SARS-CoV-2 vaccines that will be vital to ending the COVID-19 pandemic.

When employing valuable animal resources in research, particularly nonhuman primates, scientists must carefully consider the most appropriate model for the planned study. For decades, rhesus macaques - especially those of Indian origin - have been the nonhuman primate most frequently used in biomedical research, resulting in detailed genetic characterization of this species, extensively defined pedigrees, creation and maintenance of specific-pathogen-free colonies of rhesus macaques, and development of a broad range of tools for use with these animals, including specific antibodies and other reagents. These well-developed research resources have resulted in many biomedical researchers continuing to rely heavily on rhesus macaques, particularly for investigations into infectious diseases.

However, a viable alternative to rhesus macaques is needed because of the difficulty ensuring an adequate supply of these animals to sustain NIH-supported research over the past few years ${ }^{1,2}$. The increased use of another nonhuman primate in COVID-19 research could spare specific-pathogen-free Indian-origin rhesus macaques for studies of other infectious diseases.

The most viable alternative to the rhesus macaque is the baboon, whose biology closely approximates human biology ${ }^{3}$. Like humans, baboons have four immunoglobulin $\mathrm{G}$ subclasses (IgG1-4) with close genetic identity to their human counterparts ${ }^{4}$, making them excellent models for many infectious diseases, including respiratory diseases like pneumonia ${ }^{5}$, whooping $\operatorname{cough}^{6}$ and COVID-19. In addition, unlike macaques, baboons breed throughout the year with high fecundity.

In a recent study published in Nature Microbiology, researchers from the Southwest National Primate Research Center at Texas Biomedical Research Institute compared acute infection with SARS-CoV-2 in the lungs of rhesus macaques, baboons and marmosets ${ }^{7}$. The researchers recommended the continued use of the rhesus macaque as a model for developing vaccines because the animal's immune response is comparable to humans and considerable data have been accumulated using this model. Rhesus macaques, however, demonstrate mild disease symptoms, which limits their use for testing therapeutics. Baboons, on the other hand, harbor higher viral titers and have longer viremia and more pronounced lung pathology relative to macaques infected with SARS-CoV-2. Baboons therefore offer a potentially superior alternative to macaques for evaluating antiviral or immunomodulator classes of therapeutics and for understanding the comorbidities driving severe COVID-19 symptoms ${ }^{1}$.

ORIP supports well-characterized baboon resources at the Southwest National Primate Research Center and Keeling Center for Comparative Medicine and Research. The Keeling Center's Specific-Pathogen-Free Baboon Research Resource has an extensive exclusion list of 19 pathogens, making this resource ideal for studies of therapeutic interventions that require the elimination of potentially confounding results due to concurrent infections.

Infection studies have shown that some nonhuman primate species other than macaques and baboons can also develop COVID-19-like symptoms ${ }^{8-10}$. For example, a study reported that African green monkeys infected with SARS-CoV-2 had pathological lesions and disease similar to severe COVID-19 in humans ${ }^{8}$. Additional studies demonstrated that, like humans, the African green monkey model has a spectrum of responses to SARS-CoV-2 infection, from mild to severe, and thus has potential to help scientists understand the human immune response to SARS-CoV-2 and COVID-19 symptoms. ORIP provides support to the Wake Forest School of Medicine Vervet Research Colony, which maintains pedigreed, phenotyped, genotyped and genetically sequenced specific-pathogen-free African green monkeys. Although resources are limited, the NIH has begun implementing strategies and investing in the infrastructure to increase breeding of these monkey at the Vervet Research Colony. Furthermore, unlike rhesus macaques, African green monkeys can be easily 
imported to the United States from the twin-island nation of St. Kitts and Nevis, where they are free-roaming and found in significant numbers.

The NIH continues to respond to the increasing demand for nonhuman primate resources by investing in the infrastructure of existing NPRCs and primate centers that is needed to expand rhesus macaque breeding capabilities. Because the SARS-CoV-2 pandemic has significantly increased the demand for rhesus macaques, which might outstrip the supply, biomedical researchers will need to consider alternative nonhuman primate models for COVID-19 research and for other biomedical research studies.

\section{Michael C. Chang ${ }^{凶}$, Sheri Hild and Franziska Grieder}

Office of Research Infrastructure Programs, Division of Program Coordination, Planning, and Strategic Initiatives, Office of the Director, National Institutes of Health, Bethesda, Maryland, USA.

凶e-mail:changmic@mail.nih.gov

Published online: 30 March 2021

https://doi.org/10.1038/s41684-021-00755-6
References

1. Feister, A.J. et al. Nonhuman Primate Evaluation and Analysis Part 1: Analysis of Future Demand and Supply (Leidos, 2018).

2. Feister, A.J. Nonhuman Primate Evaluation and Analysis Part 2 Report of the Expert Panel Forum on Challenges in Assessing Nonhuman Primate Needs and Resources for Biomedical Research (Leidos, 2018).

3. Cox, L. A. ILAR Journal 54, 106-21 (2013).

4. Attanasio, R., Jayashankar, L., Engleman, C. N. \& Scinicariello, F. Immunogenetics 54, 556-61 (2002).

5. McFarlane, D., Wolf, R. F., McDaniel, K. A. \& White, G. L. Journals of Gerontology Series A: Biomedical Sciences and Medical Sciences 66, 1309-17 (2011).

6. Merkel, T. J. Clinical Infectious Diseases 71, 412-4 (2020).

7. Singh, D. K. et al. Nature Microbiology 6, 73-86 (2021).

8. Blair, R. et al. American Journal of Pathology 191, 274-82 (2021).

9. Rockx, B. et al. Science 29, 1012-5 (2020).

10. Lu, S. et al. Signal Transduction and Targeted Therapy 5, 157 (2020). 